\title{
Strategy for detecting off-target sites in genome-edited rice
}

Jumpei Narushima ${ }^{1}$, Shinya Kimata ${ }^{1}$, Yuh Shiwa ${ }^{2}$, Takahiro Gondo ${ }^{3}$, Satoru Akimoto ${ }^{1}$, Keisuke Soga ${ }^{1}$, Satoko Yoshiba ${ }^{1}, K_{\text {Kosuke Nakamura }}^{1, *}$, Norihito Shibata ${ }^{1}$ and Kazunari Kondo ${ }^{1, *}$

${ }^{1}$ Division of Biochemistry, National Institute of Health Sciences, Kawasaki, Kanagawa 210-9506, Japan

${ }^{2}$ Department of Molecular Microbiology, Tokyo University of Agriculture, Setagaya, Tokyo 156-8502, Japan

${ }^{3}$ Frontier Science Research Center, University of Miyazaki, Miyazaki-city, Miyazaki 889-2192, Japan

*Corresponding author: kosnakamura@nihs.go.jp, kondo@nihs.go.jp 


\begin{abstract}
Genome-editing using the CRISPR-Cas9 system can substantially accelerate crop breeding. Because off-target editing is the main problem with this system, a reliable method for comprehensively detecting off-target sites is required for the editing of food crop genomes. However, a method that accurately predicts off-target sites has not been established. In this study, we performed a SITE-Seq analysis to predict potential off-target sites. SITE-Seq is an unbiased method applicable for the in vitro detection of double-strand breaks (DSBs). To analyze SITE-Seq data, we developed a novel Galaxy system, which can perform simple and reproducible analyses without a command line operation. We conducted a SITE-Seq analysis of a rice genome modified by $O s F H 15$ gRNA-Cas9, and identified 41 DSB sites in the annotated regions. Amplicon-sequencing revealed mutations at one off-target site in the genome-edited rice. The presence of an uncommon protospacer adjacent motif (NTG PAM) likely makes this off-target site difficult to identify using in silico methods. Of the six tested programs, only CRISPRdirect predicted this off-target site, but it also predicted 6,080 off-target sites in total. These results suggest the SITE-Seq method presented herein can efficiently predict off-target sites and is useful for assessing the safety of genome-edited food.
\end{abstract}




\section{Introduction}

Genome-editing technology is a powerful tool used for plant breeding ${ }^{1}$, gene therapy ${ }^{2}$, and drug development ${ }^{3}$. More specifically, the clustered regularly interspaced short palindromic repeats (CRISPR) and CRISPR-associated protein 9 (Cas9) system has been recently applied for improving the traits of various crops, including rice, maize, and tomato ${ }^{1,4-6}$. However, site-directed nucleases, such as Cas9, cleave the target site (on-target) as well as unintended sites (off-target), which is a major concern for the genetic modification of food crops $^{7-11}$. Despite the progress made in genome-editing research, accurately predicting off-target sites with double-strand breaks (DSBs) remains difficult. Comprehensively predicting off-target sites during genome-editing is important for the subsequent assessment of food safety. Although the safety of genome-edited food must be confirmed, there is a lack of a standard protocol for predicting off-target sites.

Off-target sites are currently predicted mainly via in silico or experimental methods. The in silico methods involve a search for on-target sites based on sequence homology. To date, many in silico methods have been developed and are widely used for predicting off-target sites ${ }^{12-18}$. However, it is unclear whether these methods can detect all off-target sites, especially low-homology sites. Furthermore, thresholds for an acceptable number of mismatches and gaps have not been established. In contrast, experimental methods apply next-generation sequencing (NGS) to detect DSBs ${ }^{7-11,19-26}$. These methods can be divided primarily as in vivo ${ }^{19-22}$ and in vitro ${ }^{7,8,23-25}$ methods. Because in vivo methods detect DSBs produced by a ribonucleoprotein (RNP; guide RNA [gRNA]-Cas9 protein complex) in the intracellular environment, predictions are closer to the actual genome-editing situation. However, some off-target sites may be missed because DSBs are repaired in cells ${ }^{11}$. The in vitro methods detect DSBs produced by RNP using purified DNA. Although in vitro methods are superior to in vivo methods in terms of sensitivity and are applicable for diverse species, including animals and plants, the cut sites identified by in vitro methods include many false positives ${ }^{10,11}$.

Developing a comprehensive protocol for detecting off-target sites is essential for enhancing the application of genome-editing technology for food production. We believe in vitro methods are necessary for identifying off-target sites for genome-edited food because they are unbiased and highly sensitive. In this study, we used the selective enrichment and identification of tagged genomic DNA ends by sequencing (SITE-Seq) ${ }^{7}$, which is an unbiased in vitro method, to predict possible off-target cleavages using gRNA designed for rice (Oryza sativa L.) as a model crop. Furthermore, we developed a novel system, namely the Galaxy for Cut Site Detection program, which is run on the Docker container and used for SITE-Seq analyses. The results were compared with those obtained from in silico methods for predicting off-target sites. Additionally, the utility of SITE-Seq was verified by amplicon-sequencing. A series of analyses revealed one off-target site in genome-edited rice that was difficult to predict using in silico methods. Our data suggest that SITE-Seq is an effective method for 
predicting off-target sites in genome-edited food. Therefore, detecting off-target sites by combining in silico methods and SITE-Seq may lead to improved evaluations of the safety of genome-edited food. 


\section{Results}

The workflow in this study is presented in Figure 1. First, rice genomic DNA (gDNA) extracted from seedlings was digested with RNP under in vitro conditions for the subsequent SITE-Seq analysis. The resulting data were analyzed using the Galaxy for Cut Site Detection program we developed for detecting sites cleaved by RNP. The Galaxy system only requires a sequencing data file, a reference genome file, and a genome annotation file. Any species can be analyzed if a reference genome file is available. Next, we used the CRISPR-Cas9 system to generate genome-edited rice, after which we performed amplicon-sequencing using NGS technology to verify the mutation at the cut sites predicted by SITE-Seq. A negative control was necessary for determining whether the mutation resulted from genome-editing, endogenous single nucleotide polymorphisms, or PCR and/or NGS errors.

\section{SITE-Seq analysis on the Galaxy system}

Galaxy (https://usegalaxy.org/) is a web-based platform that enables NGS analyses with a graphical user interface ${ }^{27}$. We developed a shell script for the SITE-Seq analysis and combined it with a previously developed program for detecting cut sites $^{7}$. We then extended the pipeline to the DockerGalaxy system (Fig. 2). The pipeline was as follows: (1) SITE-Seq read data were aligned to the reference genome using Bowtie2, and the data were converted to a bam format using Samtools. (2) The aligned reads in the bam format and the reference genome file were analyzed to detect cut sites using the reported program ${ }^{7}$, and the data were exported in a bed format. (3) Details regarding the cut sites were compared with the information in the reference annotation file, after which the cut sites in annotated genes were extracted using Bedtools. (4) The extracted cut sites were refined using the awk command. Finally, the information was exported as a text file. The cut sites detected via the Galaxy for Cut Site Detection program are listed in the Supplementary Dataset.

\section{Cut sites detected by SITE-Seq}

For the SITE-Seq analysis, we focused on the OsFH15 gene. An earlier study proved that knocking out this gene via CRISPR-Cas9 genome-editing results in decreased rice grain size $^{28}$. In this study, the reported gRNA was used as the basis for editing the rice genome. The DNA libraries for the SITE-Seq analysis were prepared using rice (cv. Nipponbare) gDNA treated with three Cas9 concentrations (64, 256 , and 1,024 nM). A total of 219, 484, and 425 cut sites in the whole genome were detected following the 64, 256, and 1,024 nM Cas9 treatments, respectively (Fig. 3a, Supplementary Dataset). These results suggested that the cut sites were comprehensively detected following the treatments with 256 and 1,024 nM Cas9. The annotated regions comprised 41 cut sites. More precisely, 4 sites (two in the exon and two in the untranslated region [UTR]), 12 sites (eight in the exon, two in the intron, and two in the UTR), and 35 sites (20 in the exon, 4 in the intron, and 11 in the UTR) were detected after the 
64, 256, and 1,024 nM Cas9 treatments, respectively (Fig. 3b). Regarding these cut sites in the annotated regions, we further investigated nine sites detected at more than one Cas 9 concentration. The sites included one on-target and eight off-target sites (Fig. 3c, d and Supplementary Table S1).

To determine whether the nine sites predicted by SITE-Seq were digested by RNP, we performed a quantitative PCR (qPCR) analysis of one on-target and three off-target sites (3, 4, and 7) with many mapped reads (Fig. 3d). The qPCR data indicated the Cq values were higher for the digested samples than for the negative control (Supplementary Fig. S1), implying the template DNA quantity decreased because of the digestion by RNP. Next, we used the $\Delta$ Cq value to calculate the efficiency of the RNP cleavage. At all Cas9 concentrations, the on-target site was efficiently digested ( $>90 \%)$ (Table 1). Off-target sites 3, 4, and 7 were significantly digested (>50\%) at 1,024 nM Cas9 (Table 1). Accordingly, the sites predicted by SITE-Seq might be edited by Cas9 under in vivo conditions.

\section{Comparison between SITE-Seq and in silico programs for predicting off-target sites}

We performed an in silico analysis to predict off-target sites for OsFH15-gRNA. Moreover, we checked whether the nine cut sites detected by SITE-Seq could be predicted by the in silico analysis (Table 2). The tested programs were unable to predict off-target sites 1, 2, 5, 6, and 8, probably because of the low homology with the on-target protospacer sequence. Off-target site 3, which contains the NAG protospacer adjacent motif (PAM), was predicted by three programs (Cas-OFFinder ${ }^{13}$, CRISPRdirect ${ }^{16}$, and CRISPR-DT ${ }^{17}$ ), whereas off-target site 4 was predicted by two programs (CasOFFinder and CRISPRdirect). Off-target site 7, which contains the NTG PAM sequence, was predicted by only CRISPRdirect because this program allows mismatches in the PAM sequence. However, 6,080 candidate off-target sites were detected by CRISPRdirect, making it difficult to thoroughly analyze all of these sites (Supplementary Table S2).

\section{Validation of potential off-target sites in genome-edited rice calli}

To evaluate whether the cut sites detected by SITE-Seq would be digested by Cas9 under in vivo conditions, we produced genome-edited rice using OsFH15-gRNA. We introduced a plant expression cassette with Cas9 and gRNA into rice calli according to an Agrobacterium-mediated transformation method. Transformed calli were selected on the basis of their resistance to hygromycin B, after which they were cultured for 1 or 2 months without any regeneration. First, we performed a T7 endonuclease I (T7E1) assay to detect mutations at one on-target site and three off-target sites $(3,4$, and 7$)$. The T7E1 assay is useful for detecting heteroduplexed DNA resulting from mismatches generated by errors while DSBs are being repaired (e.g., by the non-homologous end joining pathway) ${ }^{29}$. The assay of the genome-edited samples revealed T7E1-digested fragments, indicative of genomic modifications, for the on-target site, but not for any of the off-target sites (Supplementary Fig. S2). Because of the low sensitivity of the T7E1 assay, it may not detect low-frequency mutations. Therefore, we sequenced 
amplicons to more precisely examine the nine cut sites. The amplicon-sequencing results confirmed that the genome-edited samples had various mutations, such as substitutions, deletions, or insertions, at the on-target site. Up to $10.57 \%$ of the wild-type reads were in the genome-edited samples cultured for 1 month, whereas more than $95 \%$ of the wild-type reads were in the negative control (Fig. $4 \mathrm{a}$, Supplementary Fig. S3). The percentage of wild-type reads in the genome-edited samples cultured for 2 months was less than $0.1 \%$ (Supplementary Fig. S4), indicating that the on-target site was almost completely modified under in vivo conditions. We then analyzed the eight candidate off-target sites in detail. Of three genome-edited lines cultured for 1 month, we detected a 5-nt deletion at off-target site 7 in only one line (GE1-3); the mutation was absent in the negative control sample (Fig. 4b, Supplementary Fig. S17). Regarding the other candidate off-target sites, no specific mutations were identified in the genome-edited samples (Supplementary Figures S5-S16, S19, and S20). Additionally, specific mutations were undetectable at off-target site 7 in the genome-edited samples cultured for 2 months (Supplementary Fig. S18).

\section{Assessment of the allergenicity of modified sequences}

The gRNA used in this study corresponded to exon 2 of $O s F H 15$, whereas off-target site 7 was located in the intron of Os12g0403800. Therefore, we examined the allergenicity of the sequences with a modified on-target site revealed by amplicon-sequencing using the COMPARE Database. According to the 80-mer Sliding Window FASTA Search and 8-mer FASTA Search analyses, none of the tested sequences were allergenic. 


\section{Discussion}

In this study, we conducted SITE-Seq and amplicon-sequencing analyses to investigate candidate offtarget sites in RNP-digested rice genome and in genome-edited rice calli, respectively. Although the gRNA used in this study was highly specific to the on-target site, a mutation was also detected at offtarget site 7, which contains the NTG PAM, under in vivo conditions. The digestion of a target site by RNP depends on the recognition of a PAM sequence ${ }^{30-32}$. Earlier studies revealed SpCas9 only weakly modifies genome sequences at sites containing NTG PAM ${ }^{25,32}$, but is more active at sites containing NGG or NAG PAM ${ }^{25,30-32}$. Thus, NTG PAM was considered relatively unimportant for predicting offtarget sites. However, our data suggest that a site containing NTG PAM should also be considered as a potential site digested by SpCas9 when assessing the safety of genome-edited food and human therapeutics. Additionally, SITE-Seq is an effective method for evaluating safety because it is an unbiased method that can predict potential cut sites regardless of the PAM sequence. Therefore, in addition to in silico analyses, off-target sites should also be predicted experimentally (e.g., SITE-Seq analysis).

We also developed the Galaxy for Cut Site Detection program, which enables highly reproducible analyses using any operating system. Knowledge of command line tools is unnecessary because the system operates on a web browser. Using this pipeline, cut sites in the whole genome or only in annotated regions, including exons, introns, and UTRs, can be detected. Mutations in introns reportedly lead to alternative splicing, such as exon skipping, intron retention, and alternative selection of $5^{\prime}$ or $3^{\prime}$ splice sites, resulting in the production of abnormal proteins ${ }^{33-35}$. Additionally, UTRs are important for translation, localization, and mRNA stability ${ }^{36,37}$. Thus, mutations in UTRs may cause various cellular abnormalities. Therefore, we propose that in addition to exons, the introns and UTRs should also be examined for the presence of off-target sites. The Galaxy for Cut Site Detection program is available online (https://hub.docker.com/repository/docker/nihsdnfi/galaxy-cutsite-detection).

We performed a SITE-Seq analysis to detect candidate sites for off-target DSBs induced by CRISPR-Cas9. Moreover, an amplicon-sequencing analysis was completed to confirm the candidate site mutations. Our results suggest that this strategy can effectively and efficiently identify off-target sites in genome-edited food. To address the possibility the mutations in the edited genome have biological effects, we used an allergen database to investigate whether the modified sequences revealed by amplicon-sequencing are allergenic. A similar evaluation of safety is necessary during the development of genome-edited food. We consider that the procedure developed in the current study (i.e., off-target prediction via SITE-Seq and in silico methods, amplicon sequencing, and allergen prediction) will be useful for determining the safety of genome-edited food. However, this approach will need to be refined to increase the accuracy of the predictions. 
In addition to CRISPR-Cas9, base editors are believed to be another important technique for genome engineering. Because the currently used base editors contain a catalytically impaired Cas 9 fused to a single-stranded DNA deaminase enzyme, they induce targeted point mutations without DSBs. Hence, the generation of insertions and deletions at off-target sites by base editors is likely suppressed $^{38}$, whereas single-nucleotide variants are generated throughout the genome ${ }^{39,40}$. On the basis of Digenome-seq ${ }^{23}$, experimental methods for detecting off-target genome-editing have been developed for cytosine and adenine base editors ${ }^{41,42}$. Because these methods involving Cas 9 nickase and endonuclease V or VIII produce blunt-ended DSBs as an intermediate, SITE-Seq may be a useful addition to these off-target detection methods designed for base editors. Furthermore, compared with methods based on Digenome-seq, SITE-Seq may detect off-target sites with a lower sequence coverage because it enriches DSBs using biotinylated oligonucleotides. In future studies, we will improve the Galaxy for Cut Site Detection program so that it can detect off-target sites resulting from base editing. 


\section{Methods}

\section{Plant materials and callus induction}

Rice (Oryza sativa L. ssp. japonica cv. Nipponbare; JP: 222430) seeds were provided by the Genetic Resources Center of the National Agriculture and Food Research Organization in Japan. To extract DNA from the wild-type rice used for the SITE-Seq analysis, surface-sterilized seeds were germinated on filter paper moistened with sterile distilled water, after which they were transferred to Murashige and Skoog medium ${ }^{43}$ supplemented with myoinositol $(10 \mathrm{mg} / \mathrm{L})$, glycine $(200 \mu \mathrm{g} / \mathrm{L})$, nicotinic acid $(50 \mu \mathrm{g} / \mathrm{L})$, pyridoxine hydrochloride $(50 \mu \mathrm{g} / \mathrm{L})$, thiamine hydrochloride $(10 \mu \mathrm{g} / \mathrm{L}), \mathrm{pH} 5.8$, and 3\% sucrose. Seedlings were incubated at $28{ }^{\circ} \mathrm{C}$ with an 8 -h light/16-h dark photoperiod. To generate rice calli, seeds were surface-sterilized and placed on N6 medium ${ }^{44}$ supplemented with 2,4dichlorophenoxyacetic acid $(2 \mathrm{mg} / \mathrm{L})$, proline $(10 \mathrm{mM})$, casein hydrolysate $(300 \mathrm{mg} / \mathrm{L})$, sucrose $(30$ $\mathrm{g} / \mathrm{L}$ ), and Gelrite (3 g/L) (N6D medium). Calli were induced for 3 weeks. Small, vigorously dividing calli were subcultured at 14-day intervals. The calli were incubated at $28{ }^{\circ} \mathrm{C}$ in darkness and then transformed.

\section{Construction of the CRISPR-Cas9-related vector and Agrobacterium-mediated transformation}

A vector based on pRGEB32 (Addgene, Watertown, MA, USA, plasmid \#63142) ${ }^{45}$ was used for genome-editing. The pair of oligonucleotides used for introducing the gRNA cassette into the vector is listed in Supplementary Table S3. The constructed vector was transferred into A. tumefaciens strain EHA105 cells, which were then used to transform rice calli as described by Ozawa ${ }^{46}$. Negative control calli were transformed with the empty pRGEB32 vector (i.e., without the gRNA cassette). After a 21day infection, transformed calli were selected on medium containing $50 \mathrm{mg} / \mathrm{L}$ hygromycin B. Independent lines were subcultured on fresh medium at 14-day intervals. The transformed callus lines were cultured for 1 or 2 months and then used for the genome-editing analysis.

\section{SITE-Seq analysis}

The SITE-Seq analysis was performed using the MiSeq system and MiSeq Reagent Kit (version 3) (150 cycles) (Illumina, Inc., San Diego, CA, USA) to produce $1 \times 151$-bp single-end reads. Specific details regarding the methods used are provided in the Supplementary Methods. The oligonucleotides used for the SITE-Seq analysis are listed in Supplementary Table S3.

\section{Galaxy for Cut Site Detection program}

Referring to a published report ${ }^{7}$, we wrote a novel shell script to analyze the SITE-Seq data (see Supplementary Script). It includes an additional function that extracts cut sites in annotated regions. Read quality was checked using FastQC (version 0.11.9), after which the reads were aligned to the 
reference genome using Bowtie $2^{47}$ (version 2-4.2). The sam file for the aligned reads was converted to a bam file before the sorting and indexing step using Samtools ${ }^{48}$ (version 1.12). The sorted bam file and the original python program described in SITE-Seq Supplementary Software ${ }^{7}$ were used for detecting cut sites. The resulting cut site data were converted to a bed file, after which cut sites within annotated regions were called using Bedtools ${ }^{49}$ (version 2.30.0). Furthermore, we built a custom Galaxy for Cut Site Detection program, which runs on a Docker container, from the developed shell script. The IRGSP-1.0 reference genome and genome annotation files were downloaded from RAPDB (https://rapdb.dna.affrc.go.jp/download/irgsp1.html) in November 2018. The cut sites detected by the SITE-Seq analysis are listed in the Supplementary Dataset.

\section{Prediction of off-target sites using in silico methods}

The following programs, which are applicable for analyzing the rice reference genome, were selected for predicting off-target sites: Cas-OFFinder ${ }^{13}$ (http://www.rgenome.net/cas-offinder/), CHOPCHOP $^{14}$ (https://chopchop.cbu.uib.no/), CRISPOR ${ }^{15}$ (http://crispor.tefor.net/crispor.py), CRISPRdirect ${ }^{16}$ (https://crispr.dbcls.jp/), CRISPR-DT ${ }^{17}$ (http://bioinfolab.miamioh.edu/CRISPRDT/), and CRISPR-P $2.0^{18}$ (http://crispr.hzau.edu.cn/CRISPR2/). The off-target sites for SpCas9 (both NGG and NRG PAM) were predicted using default parameters.

\section{qPCR analysis}

The qPCR analysis was performed using the LightCycler 480 System II (Roche Diagnostics, Rotkreuz, Switzerland) to evaluate RNP cleavage efficiency. The RNP digestion was completed in a $25-\mu \mathrm{L}$ reaction mixture containing recombinant SpCas9 (Themo Fisher Scientific, Waltham, MA, USA), gRNA, and $1.5 \mu \mathrm{g}$ rice gDNA. The final Cas9 concentrations were 64,256 , and 1,024 $\mathrm{nM}$. The method for synthesizing gRNA is described in the Supplementary Methods and details regarding the oligonucleotides used are listed in Supplementary Table S3. The digestion by RNP was performed at $37{ }^{\circ} \mathrm{C}$ for $16 \mathrm{~h}$, after which $44 \mu \mathrm{g}$ RNase A (Nippon Gene Co., Ltd., Tokyo, Japan) was added to the mixture, which was then incubated at $37^{\circ} \mathrm{C}$ for $20 \mathrm{~min}$. The reaction was terminated by adding $5 \mu \mathrm{g}$ protease $\mathrm{K}$ (Wako) and incubating the mixture at $55^{\circ} \mathrm{C}$ for $20 \mathrm{~min}$. Finally, protease $\mathrm{K}$ was deactivated via an incubation at $95{ }^{\circ} \mathrm{C}$ for $10 \mathrm{~min}$. For the negative control, water was added instead of gRNA. The qPCR was performed in a $25-\mu \mathrm{L}$ reaction mixture that included $12.5 \mu \mathrm{L}$ THUNDERBIRD SYBR qPCR Mix (Toyobo Co., Ltd., Osaka, Japan), $0.5 \mu \mathrm{M}$ each primer, and $5 \mathrm{ng}$ RNP-digested DNA. The qPCR primers are listed in Supplementary Table S3. The $\triangle \mathrm{Cq}$ values were calculated by subtracting the $\mathrm{Cq}$ value of the negative control from the $\mathrm{Cq}$ value of the genome-edited sample. Cleavage efficiency $(E)$ was calculated using the following equation:

$$
E(\%)=\left(1-\frac{1}{2^{\Delta \mathrm{Cq}}}\right) \times 100
$$




\section{T7 endonuclease I assay}

The on-target and off-target sites detected by SITE-Seq were amplified from genome-edited rice DNA using specific primers (Supplementary Table S3). The PCR products were analyzed by $2 \%$ agarose gel electrophoresis and purified using the NucleoSpin Gel and PCR Clean-up kit (Macherey-Nagel, GmbH \& Co. KG, Dueren, Germany). Approximately 200 ng DNA products were mixed with NEBuffer $2\left(50 \mathrm{mM} \mathrm{NaCl}, 10 \mathrm{mM}\right.$ Tris-HCl, $10 \mathrm{mM} \mathrm{MgCl}_{2}$, and $1 \mathrm{mM}$ dithiothreitol, $\mathrm{pH}$ 7.9) and incubated at $95{ }^{\circ} \mathrm{C}$ for $5 \mathrm{~min}$ before gradually decreasing the temperature to $25^{\circ} \mathrm{C}\left(0.1{ }^{\circ} \mathrm{C} / \mathrm{s}\right)$. After adding 2.5 U T7 endonuclease I (New England Biolabs Inc., Ipswich, MA, USA), the mixture was incubated at $25{ }^{\circ} \mathrm{C}$ for $15 \mathrm{~min}$ before $1.5 \mu \mathrm{L} 0.25 \mathrm{M}$ EDTA was added to terminate the reaction. The reaction products were analyzed by $2 \%$ agarose gel electrophoresis.

\section{Amplicon-sequencing}

Nine cut sites detected by SITE-Seq were amplified from genome-edited rice callus DNA using specific primers (Supplementary Table S3). The DNA libraries were prepared as described in the Supplementary Methods. Amplicons were sequenced using the iSeq 100 system and the iSeq $100 \mathrm{il}$ Reagent (300 Cycles) (Illumina) to generate $2 \times 151$-bp paired-end reads. The CRISPResso2 ${ }^{50}$ program (version 2.0.31; https://github.com/pinellolab/CRISPResso2) was used to analyze the mutation rate greater than $0.1 \%$.

\section{Allergenicity prediction}

The allergenicity of the modified sequences revealed by amplicon-sequencing was predicted using the COMPARE 2020 database released on January 29, 2020 (https://comparedatabase.org/). More specifically, the allergenicity was assessed according to the 80-mer Sliding Window FASTA Search and the 8-mer FASTA Search programs. 


\section{References}

1. Ku, H. K. \& Ha, S. H. Improving Nutritional and Functional Quality by Genome Editing of Crops: Status and Perspectives. Front. Plant Sci. 11, 577313; 10.3389/fpls.2020.577313 (2020).

2. Li, H. et al. Applications of genome editing technology in the targeted therapy of human diseases: mechanisms, advances and prospects. Signal Transduct. Target Ther. 5, 1; 10.1038/s41392-0190089-y (2020).

3. Potekhina, E. S. et al. Drug Screening with Genetically Encoded Fluorescent Sensors: Today and Tomorrow. Int. J. Mol. Sci. 22; 10.3390/ijms22010148 (2020).

4. Sun, Y. et al. Generation of High-Amylose Rice through CRISPR/Cas9-Mediated Targeted Mutagenesis of Starch Branching Enzymes. Front. Plant Sci. 8, 298; 10.3389/fpls.2017.00298 (2017).

5. Waltz, E. CRISPR-edited crops free to enter market, skip regulation. Nat. Biotechnol. 34, 582 (2016).

6. Nonaka, S., Arai, C., Takayama, M., Matsukura, C. \& Ezura, H. Efficient increase of aminobutyric acid (GABA) content in tomato fruits by targeted mutagenesis. Sci. Rep. 7, 7057; 10.1038/s41598-017-06400-y (2017).

7. Cameron, P. et al. Mapping the genomic landscape of CRISPR-Cas9 cleavage. Nat. Methods. 14, 600-606 (2017).

8. Young, J. et al. CRISPR-Cas9 Editing in Maize: Systematic Evaluation of Off-target Activity and Its Relevance in Crop Improvement. Sci. Rep. 9, 6729; 10.1038/s41598-019-43141-6 (2019).

9. Naeem, M., Majeed, S., Hoque, M. Z. \& Ahmad, I. Latest Developed Strategies to Minimize the Off-Target Effects in CRISPR-Cas-Mediated Genome Editing. Cells. 9, 1608; 10.3390/cells9071608 (2020).

10. Stortz, F. \& Minary, P. crisprSQL: a novel database platform for CRISPR/Cas off-target cleavage assays. Nucleic Acids Res. 49, D855-D861; 10.1093/nar/gkaa885 (2021).

11. Bao, X. R., Pan, Y., Lee, C. M., Davis, T. H. \& Bao, G. Tools for experimental and computational analyses of off-target editing by programmable nucleases. Nat. Protoc. 16, 10-26 (2021).

12. Cui, Y., Xu, J., Cheng, M., Liao, X. \& Peng, S. Review of CRISPR/Cas9 sgRNA Design Tools. Interdiscip. Sci. 10, 455-465 (2018).

13. Bae, S., Park, J. \& Kim, J. S. Cas-OFFinder: a fast and versatile algorithm that searches for potential off-target sites of Cas9 RNA-guided endonucleases. Bioinformatics. 30, 1473-1475 (2014).

14. Labun, K. et al. CHOPCHOP v3: expanding the CRISPR web toolbox beyond genome editing. Nucleic Acids Res. 47, W171-W174; 10.1093/nar/gkz365 (2019).

15. Concordet, J. P. \& Haeussler, M. CRISPOR: intuitive guide selection for CRISPR/Cas9 genome 
editing experiments and screens. Nucleic Acids Res. 46, W242-W245; 10.1093/nar/gky354 (2018).

16. Naito, Y., Hino, K., Bono, H. \& Ui-Tei, K. CRISPRdirect: software for designing CRISPR/Cas guide RNA with reduced off-target sites. Bioinformatics. 31, 1120-1123 (2015).

17. Zhu, H. \& Liang, C. CRISPR-DT: designing gRNAs for the CRISPR-Cpf1 system with improved target efficiency and specificity. Bioinformatics. 35, 2783-2789 (2019).

18. Liu, H. et al. CRISPR-P 2.0: An Improved CRISPR-Cas9 Tool for Genome Editing in Plants. Mol. Plant. 10, 530-532 (2017).

19. Crosetto, N. et al. Nucleotide-resolution DNA double-strand break mapping by next-generation sequencing. Nat. Methods. 10, 361-365 (2013).

20. Tsai, S. Q. et al. GUIDE-seq enables genome-wide profiling of off-target cleavage by CRISPRCas nucleases. Nat. Biotechnol. 33, 187-197 (2015).

21. Ortinski, P. I., O'Donovan, B., Dong, X. \& Kantor, B. Integrase-Deficient Lentiviral Vector as an All-in-One Platform for Highly Efficient CRISPR/Cas9-Mediated Gene Editing. Mol. Ther. Methods Clin. Dev. 5, 153-164 (2017).

22. Wienert, B. et al. Unbiased detection of CRISPR off-targets in vivo using DISCOVER-Seq. Science. 364, 286-289 (2019).

23. Kim, D. et al. Digenome-seq: genome-wide profiling of CRISPR-Cas9 off-target effects in human cells. Nat. Methods. 12, 237-243 (2015).

24. Tsai, S. Q. et al. CIRCLE-seq: a highly sensitive in vitro screen for genome-wide CRISPR-Cas9 nuclease off-targets. Nat. Methods. 14, 607-614 (2017).

25. Lazzarotto, C. R. et al. CHANGE-seq reveals genetic and epigenetic effects on CRISPR-Cas9 genome-wide activity. Nat. Biotechnol. 38, 1317-1327 (2020).

26. Akcakaya, P. et al. In vivo CRISPR editing with no detectable genome-wide off-target mutations. Nature. 561, 416-419 (2018).

27. Goecks, J., Nekrutenko, A., Taylor, J. \& Galaxy, T. Galaxy: a comprehensive approach for supporting accessible, reproducible, and transparent computational research in the life sciences. Genome Biol. 11, R86; 10.1186/gb-2010-11-8-r86 (2010).

28. Sun, T., Li, S. \& Ren, H. OsFH15, a class I formin, interacts with microfilaments and microtubules to regulate grain size via affecting cell expansion in rice. Sci. Rep. 7, 6538; 10.1038/s41598-01706431-5 (2017).

29. Sentmanat, M. F., Peters, S. T., Florian, C. P., Connelly, J. P. \& Pruett-Miller, S. M. A Survey of Validation Strategies for CRISPR-Cas9 Editing. Sci. Rep. 8, 888; 10.1038/s41598-018-19441-8 (2018).

30. Jinek, M. et al. A programmable dual-RNA-guided DNA endonuclease in adaptive bacterial immunity. Science. 337, 816-821 (2012).

31. Hsu, P. D. et al. DNA targeting specificity of RNA-guided Cas9 nucleases. Nat. Biotechnol. 31, 
827-832 (2013).

32. Chatterjee, P., Jakimo, N. \& Jacobson, J. M. Minimal PAM specificity of a highly similar SpCas9 ortholog. Sci. Adv. 4, eaau0766; 10.1126/sciadv.aau0766 (2018).

33. Syed, N. H., Kalyna, M., Marquez, Y., Barta, A. \& Brown, J. W. Alternative splicing in plants-coming of age. Trends Plant Sci. 17, 616-623 (2012).

34. Anna, A. \& Monika, G. Splicing mutations in human genetic disorders: examples, detection, and confirmation. J. Appl. Genet. 59, 253-268 (2018).

35. Bryen, S. J. et al. Pathogenic Abnormal Splicing Due to Intronic Deletions that Induce Biophysical Space Constraint for Spliceosome Assembly. Am. J. Hum. Genet. 105, 573-587 (2019).

36. Leppek, K., Das, R. \& Barna, M. Functional 5' UTR mRNA structures in eukaryotic translation regulation and how to find them. Nat. Rev. Mol. Cell Biol. 19, 158-174 (2018).

37. Mayr, C. Regulation by 3'-Untranslated Regions. Annu. Rev. Genet. 51, 171-194 (2017).

38. Eid, A., Alshareef, S. \& Mahfouz, M. M. CRISPR base editors: genome editing without doublestranded breaks. Biochem J. 475, 1955-1964 (2018).

39. Jin, S. et al. Cytosine, but not adenine, base editors induce genome-wide off-target mutations in rice. Science. 364, 292-295 (2019).

40. Zuo, E. et al. Cytosine base editor generates substantial off-target single-nucleotide variants in mouse embryos. Science. 364, 289-292 (2019).

41. Kim, D. et al. Genome-wide target specificities of CRISPR RNA-guided programmable deaminases. Nat Biotechnol. 35, 475-480 (2017).

42. Kim, D., Kim, D. E., Lee, G., Cho, S. I. \& Kim, J. S. Genome-wide target specificity of CRISPR RNA-guided adenine base editors. Nat Biotechnol. 37, 430-435 (2019).

43. Murashige, T. \& Skoog, F. A revised medium for rapid growth and bioassays with tobacco tissue cultures. Physiologia Plantarum. 15, 473-497 (1962).

44. Chu, C. et al. Establishment of an efficient medium for anther culture of rice through comparative experiments on the nitrogen sources. Scientia Sinica. 18, 223-231 (1975).

45. Xie, K., Minkenberg, B. \& Yang, Y. Boosting CRISPR/Cas9 multiplex editing capability with the endogenous tRNA-processing system. Proc. Natl. Acad. Sci. USA. 112, 3570-3575 (2015).

46. Ozawa, K. Establishment of a high efficiency Agrobacterium-mediated transformation system of rice (Oryza sativa L.). Plant Sci. 176, 522-527 (2009).

47. Langmead, B. \& Salzberg, S. L. Fast gapped-read alignment with Bowtie 2. Nat. Methods. 9, 357359 (2012).

48. Li, H. et al. The Sequence Alignment/Map format and SAMtools. Bioinformatics. 25, 2078-2079 (2009).

49. Quinlan, A. R. \& Hall, I. M. BEDTools: a flexible suite of utilities for comparing genomic features. Bioinformatics. 26, 841-842 (2010). 
bioRxiv preprint doi: https://doi.org/10.1101/2021.05.28.446070; this version posted May 29, 2021. The copyright holder for this preprint (which was not certified by peer review) is the author/funder. All rights reserved. No reuse allowed without permission.

50. Clement, K. et al. CRISPResso2 provides accurate and rapid genome editing sequence analysis. Nat. Biotechnol. 37, 224-226 (2019). 


\section{Acknowledgments}

This study was supported in part by the Ministry of Health, Labor and Welfare (H30-Syokuhin-Ippan002 and 21KA1002 to K.K.), and Japan Society for the Promotion of Science and the Ministry of Education, Culture, Sports, Science and Technology (JSPS/MEXT KAKENHI Grants Number JP20K05901 to K.S., JP21K06075 to N.S., and JP21H02469 to K.K.). We thank Edanz (https://jp.edanz.com/ac) for editing a draft of this manuscript.

\section{Author contributions statement}

J.N., S.K., K.N., and K.K. designed the experiments. J.N., S.K., and K.N. performed the experiments. T.G. performed the rice transformations. Y.S. and S.A. developed the Galaxy for Cut Site Detection program. J.N. analyzed the data. J.N., N.S., and K.K. wrote the manuscript. All authors reviewed the manuscript.

\section{Data availability}

All data generated or analyzed during this study are included in this published article and its Supplementary Information files. Nucleotide sequence data are available in the DNA Data Bank of Japan Sequence Read Archive under accession number PRJDB11507.

\section{Ethics declarations}

\section{Competing interests}

The authors declare no competing interests.

\section{Additional information}

\section{Supplementary information}

Supplementary Figures S1-S20 
bioRxiv preprint doi: https://doi.org/10.1101/2021.05.28.446070; this version posted May 29, 2021. The copyright holder for this preprint (which was not certified by peer review) is the author/funder. All rights reserved. No reuse allowed without permission.

\section{Supplementary Tables S1-S3}

Supplementary Methods

Supplementary Script

Supplementary Dataset 


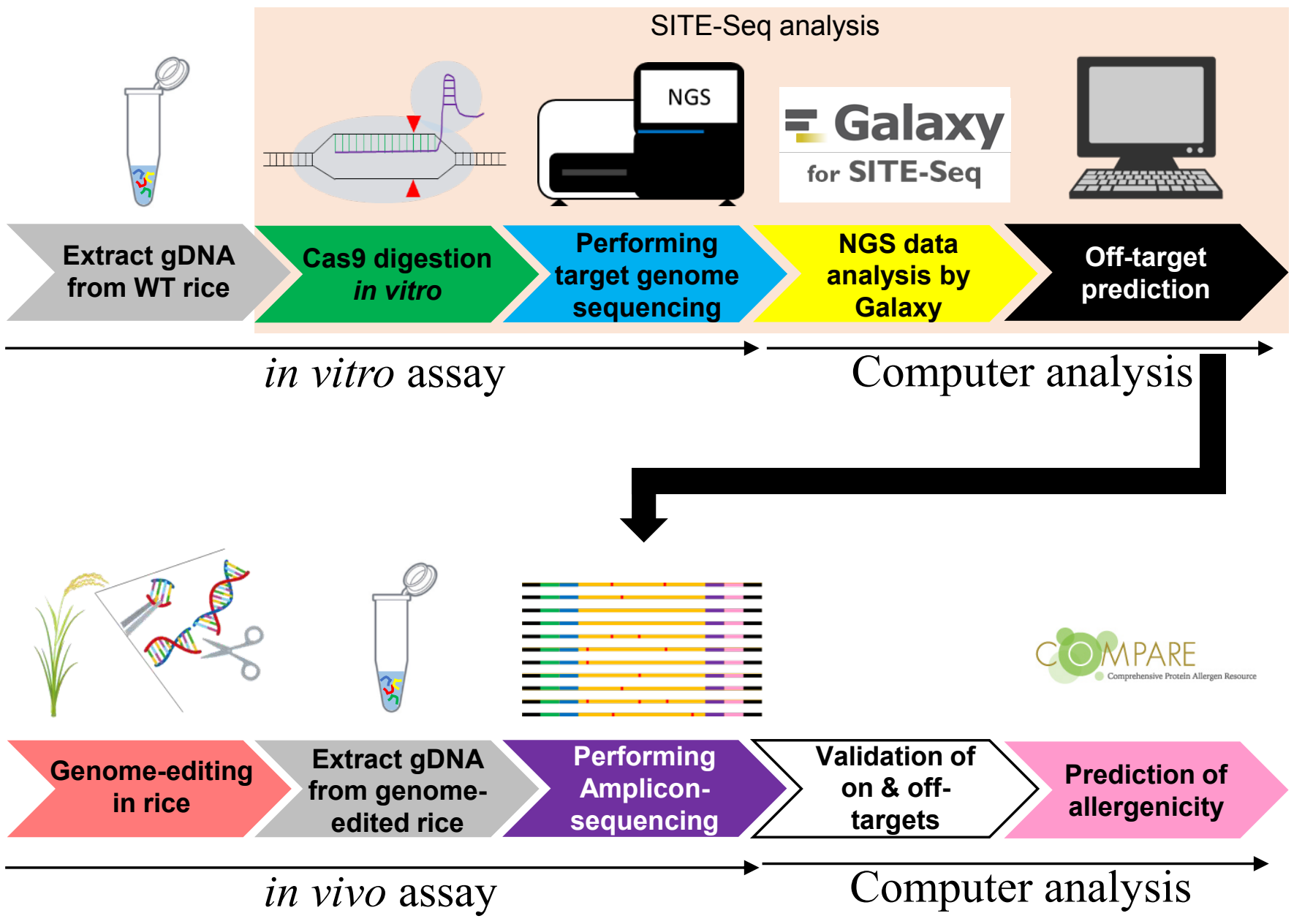

Figure 1. Study workflow 
Tools

search tools $\boldsymbol{\otimes}$

Inputs

Data Managers

Get Data

Cut Site Detection

FASTQ Quality Control

Mapping

Bedtools

Text Manipulation

Workflow
SITE-Seq

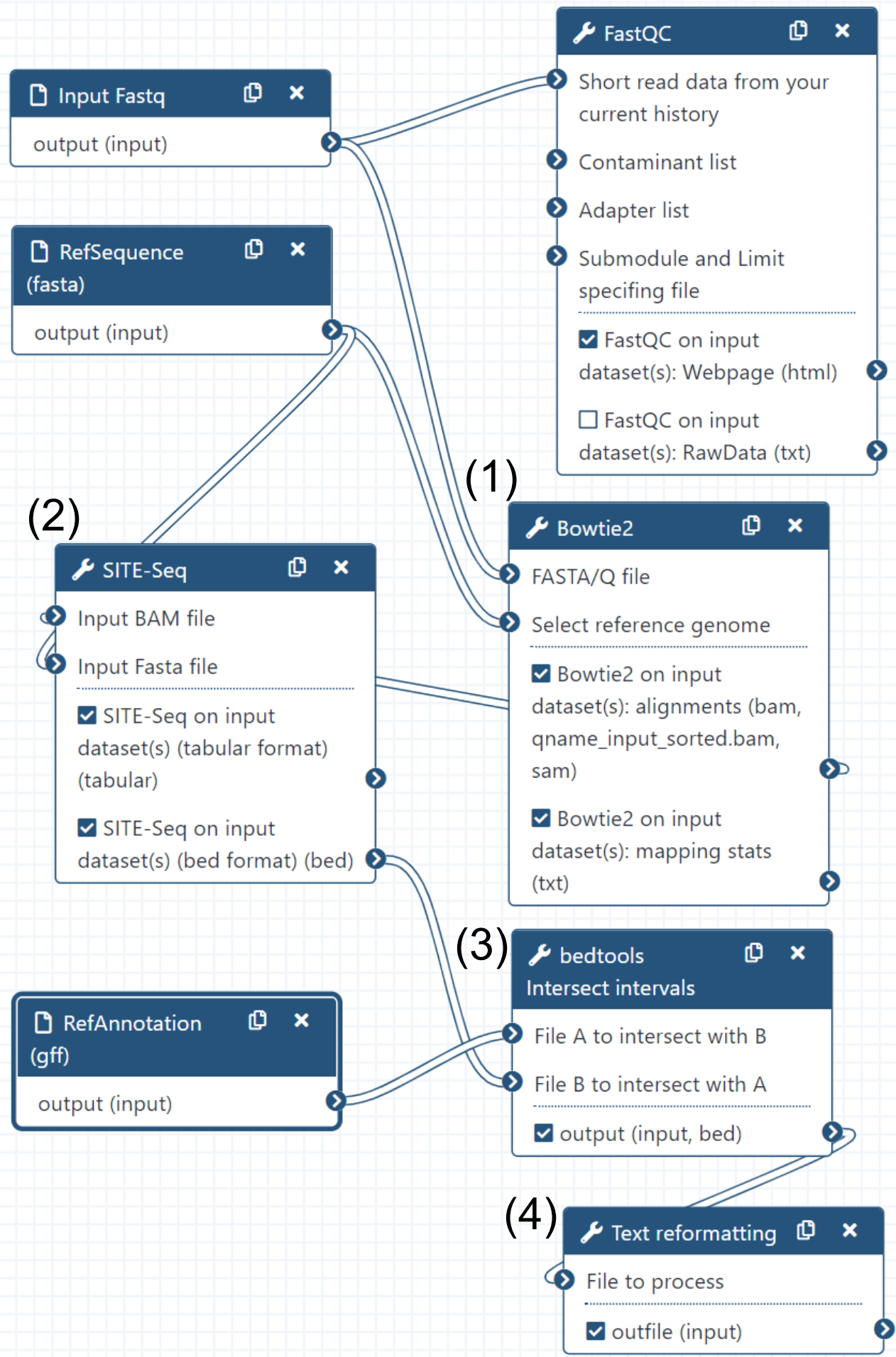

Figure 2. Galaxy for Cut Site Detection pipline. 
a

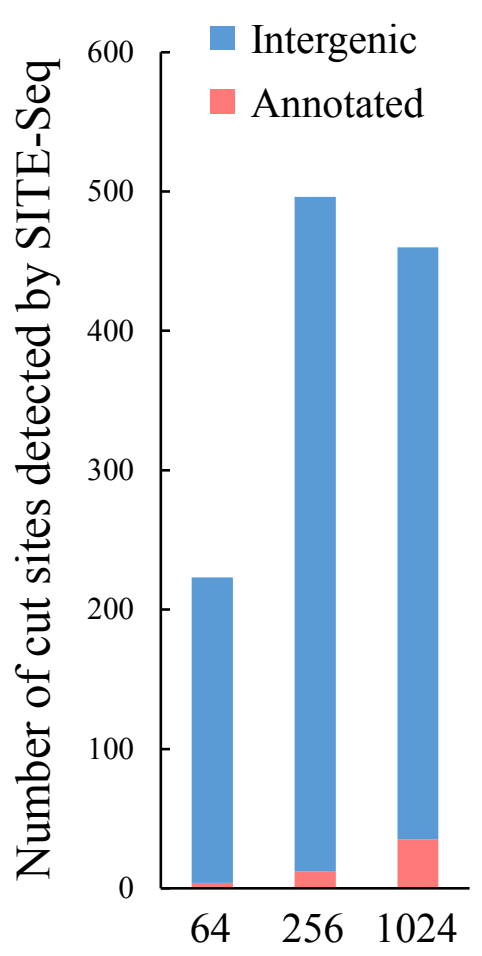

Concentration of Cas9 (nM) b

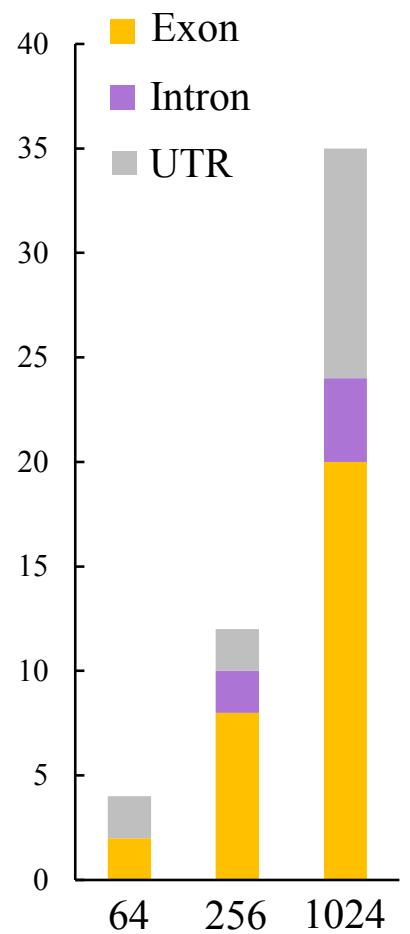

Concentration of

Cas9 (nM) c

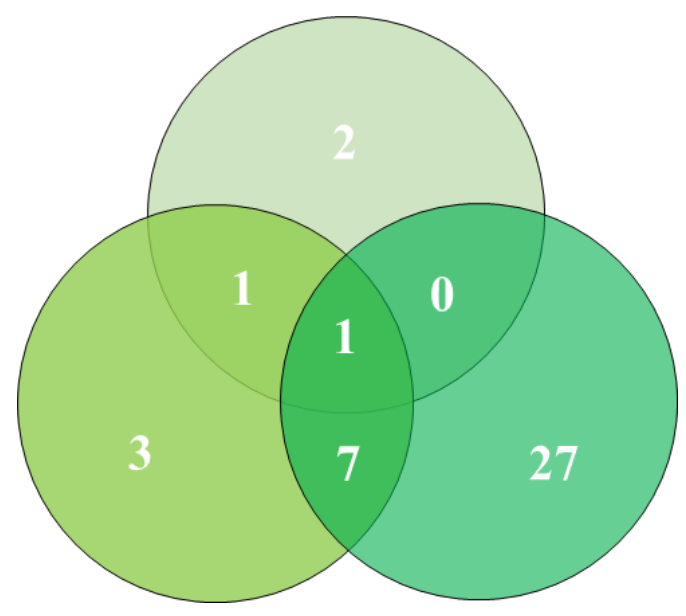

$64 \mathrm{nM}$

$256 \mathrm{nM}$

\begin{tabular}{|c|c|c|c|c|}
\hline \multirow{2}{*}{\multicolumn{2}{|c|}{ Locus }} & \multicolumn{3}{|c|}{$\begin{array}{c}\text { Number of reads mapped in } \\
\text { SITE-Seq analysis }\end{array}$} \\
\hline & & \multirow{2}{*}{$\begin{array}{c}64 \mathrm{nM} \\
39\end{array}$} & \multirow{2}{*}{$\frac{256 \mathrm{nM}}{138}$} & \multirow{2}{*}{$\frac{1024 \mathrm{nM}}{110}$} \\
\hline On-target & $\begin{array}{l}\text { chr9:20181243 } \\
\text { (Exon) }\end{array}$ & & & \\
\hline Off-target 1 & $\begin{array}{l}\text { chr1:18688154 } \\
\text { (Exon) }\end{array}$ & n.d. & 17 & 14 \\
\hline Off-target 2 & $\begin{array}{l}\text { chr1:33534133 } \\
\text { (Exon) }\end{array}$ & n.d. & 34 & 28 \\
\hline Off-target 3 & $\begin{array}{l}\text { chr1:39263530 } \\
\text { (Exon) }\end{array}$ & n.d. & 79 & 274 \\
\hline Off-target 4 & $\begin{array}{l}\text { chr5:18020045 } \\
\text { (3' UTR) }\end{array}$ & n.d. & 229 & 487 \\
\hline Off-target 5 & $\begin{array}{l}\text { chr6:15796609 } \\
\text { (Intron) }\end{array}$ & n.d. & 7 & 7 \\
\hline Off-target 6 & $\begin{array}{l}\text { chr10:10862382 } \\
\text { (Exon) }\end{array}$ & n.d. & 33 & 30 \\
\hline Off-target 7 & $\begin{array}{l}\text { chr12:12080780 } \\
\text { (Intron) }\end{array}$ & n.d. & 233 & 473 \\
\hline Off-target 8 & $\begin{array}{l}\text { chr12:20542434 } \\
\text { (3' UTR) }\end{array}$ & 14 & 7 & n.d. \\
\hline
\end{tabular}


Figure 3. Detection of cut sites by SITE-Seq. (a) Bar plot of the number of cut sites detected by SITESeq. (b) Bar plot of the number of cut sites in (a) that are in annotated genome regions. (c) Comparison of the number of cut sites in annotated regions detected at various Cas 9 concentrations. The on-target site was detected at all Cas9 concentrations. (d) Nine cut sites detected at multiple Cas9 concentrations; n.d., not detected. 
A G C A T C C A A G A A T G A G T C A A G G A G G C A A T G C A Reference

A G C A T C C A A G A A T G G A - T C A A G G A G G C A A A T G C A 26.62\% (15130 reads) A G C A T C C A A G A A T G G A G T T C A A G G A G G C A A A T G C $24.08 \%$ (13685 reads) A G C A T C C A A G A A T - - - C A A G G A G G C A A A T G C A $13.09 \%(7438$ reads) A G C A T C C A A G A A T G G A G G T C A A G G A G G C A A A T G C $5.80 \%$ (3295 reads) A G C A T C C A A G A A T G G A G . - ..... G C A A A T G C A $4.52 \%(2568$ reads) $\begin{array}{lllllllllllllllll}- & -1\end{array}$ A G C A T C C A A G A A T G G - T C A A G G A G G C A A A T G C A $3.99 \%(2267$ reads)

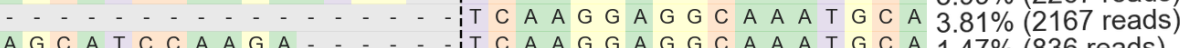
A G C A T C C A A G A ....... T C A A G G A G G C A A A T G C A $1.47 \%$ (836 reads) A G C A T C C A A G A A T G G A G T T T C A A G G A G G C A A A T G $1.27 \%$ (724 reads) - - - - - - - - - - - - - - A G C A T C C A A G A A T G G A G A T T C A A G G A G G C A A A T G $0.67 \%$ (378 reads) A G C A T C C A A G A A T G G A G T T C A A G G A G G G A A A T G C $0.31 \%(177$ reads) A G C A T C C A A G A A A G G A - T C A A G G A G G C A A A T G C A $0.29 \%$ (165 reads) A G C A T C C A A G A A T G G A - T C A A G G A G G G A A A T G C A $0.29 \%$ (165 reads)

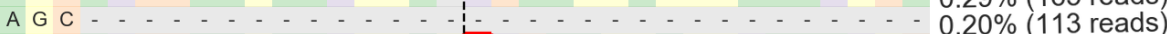
A G C A T C C A A G A A T G G A G T T C A A G G A G G T A A A T G C $0.19 \%$ (108 reads) A G C A T C C A A G A A T G G A T C A A G G A G G T A A A T G C A $0.17 \%$ (94 reads) A G C A T C C A A G A A T - - . C A A G G A G G G A A A T G C A $0.12 \%$ (70 reads)

\section{PAM Protospacer}

b

A A T T T G A T A A C AGTGACTCCATTCT TGTATGAT Reference

A A T T T G A T A T A C A G T G A A C T C C A T T C T T G T A T G A T 56.06\% (25763 reads) A A T T T G A T A T A C A G T G A A C T C C A T T C T T G A A T G A T $13.44 \%$ (6177 reads) A A T T T G A T A T A C A G T G AIC T C C A T T C T T G C A T G A T $384 \%$ (1763 reads) A A T T T G A T A T A C A G T G A C T C C A T T C A T G T A T G A T $3.54 \%$ (1628 reads) A A T T T G A T A T A C A G T G A C T C C A T T C T T A T A T G A T $3.33 \%$ (1531 reads) A A T T T G A T A T A C A G T G A C T C C A T T C C T G T A T G A T $2.81 \%$ (1293 reads)

A A T T T G A T A T A C A G T G A C T C C A T T C A T G A A T G A T $2.16 \%$ (994 reads) A A T T T G A T A T A C A G T G A I C T C C A T T C T A G T A T G A T $1.39 \%$ (640 reads) A A T T T G A T A T A C A G T G A C T C C A T T C T T A A A T G A T $1.31 \%$ (600 reads) A A T T T G A T A T A C A G T G AIC T C C A T T C T C G T A T G A T $0.99 \%$ (455 reads) A A T T T G A T A T A C A G T G A C T C C A T T C T T G G A T G A T $0.91 \%$ (416 reads) A A T T T G A T A T A C - ..... C T C C A T T C T T G T A T G A T $0.61 \%$ (282 reads) A A T T T G A T A T A C A G T G AIC T C C A T T C C T G C A T G A T $0.55 \%$ (251 reads) A A T T T G A T A T A C A G T G A C T C C A T T C T A G A A T G A T $0.54 \%$ (250 reads) A A T T T G A T A T A C A G T G A C T C C A T T C A T A T A T G A T $0.45 \%$ (206 reads) A A T T T G A T A T A C A G T G A C T C C A T T C T T G T T T G A T $0.44 \%$ (202 reads) A A T T T G A T A T A C A G T G A C T C C A T T C A T A A A T G A T $0.28 \%$ (130 reads) A A T T T G A T A T A C A G T G A C T C C A T T C T A A T A T G A T $0.26 \%$ (120 reads) A A T T T G A T A T A C A G T G A C T C C A T T C C T G A A T G A T $0.23 \%$ (106 reads) A A T T T G A T A T A C A G T G A C T C C A T T C T T G T A A G A T $0.23 \%$ (106 reads) A A T T T G A T A T A C A G T G A I C T C C A T T C T T C T A T G A T $0.22 \%$ (103 reads) A A T T T G A T A T A C A G T G ATC T C C A T T C A A G T A T G A T $0.21 \%$ (97 reads) A A T T T G A T A T A C A G T G A C T C C A T T C T C A T A T G A T $0.20 \%$ (93 reads) A A T T T G A T A T A C A G T G A C T C C A T T C T T A G A T G A T $0.17 \%$ (77 reads) A A T T T G A T A T A C A G T G A C T C C A T T C T T A C C A T G A T $0.15 \%$ (67 reads) A A T T T G A T A T A A C A G T G A C T C C A T T C C T G G A T G A T $0.13 \%$ (61 reads) A A T T T G A T A T A C A G T G A C T C C A T T C T A A A A T G A T $0.13 \%$ (60 reads) A A T T T G A T A T A C A G T G A C T C C A T T C A A G A A T G A T $0.13 \%$ (59 reads) A A T T T G A T A T A C A G T G A C T C C A T T C T C G A A T G A T $0.12 \%$ (56 reads) A A T T T G A T A T A C - .... C T C C A T T C T T G A A T G A T $0.12 \%$ (56 reads) A A T T T T G A T A A T A C A G T G A C T C C A T T C T T G A T T T G A T $0.12 \%$ (56 reads) A A T T T G A T A T A C A G T G A C T C C A T T C T C G C A T G A T $0.11 \%$ (52 reads)

\begin{tabular}{|cl|}
\hline bold & Substitutions \\
$\square$ & Insertions \\
- & Deletions \\
----- & Predicted cleavage position
\end{tabular}


Figure 4. Amplicon-sequencing analysis of the cut sites detected by SITE-Seq. Amplicons were sequenced for the genome-edited and negative control rice calli cultivated for 1 month $(\mathrm{n}=3)$. Regarding the genome-edited samples, the on-target site (a) and off-target site 7 (b), which had $>0.1 \%$ mutations, are presented. The read counts and rates are provided for each sequence. The presented data are from the same line (GE1-3). Arrows indicate a 5-nt deletion, which was not detected in the negative control (see Supplementary Fig. S17). 
Table 1. RNP cleavage efficiency for on-target and off-target sites.

\begin{tabular}{|c|c|c|c|c|c|c|c|c|c|c|c|c|c|c|c|c|}
\hline Target & & On-t & arget & & & Off-t & rget 3 & & & Off-t & rget 4 & & & Off-t: & rget 7 & \\
\hline \multirow{2}{*}{ Sample } & \multicolumn{4}{|c|}{ Cas9 concentration (nM) } & \multicolumn{4}{|c|}{ Cas9 concentration (nM) } & \multicolumn{4}{|c|}{ Cas9 concentration (nM) } & \multicolumn{4}{|c|}{ Cas9 concentration (nM) } \\
\hline & $\mathrm{NC}$ & 64 & 256 & 1,024 & $\mathrm{NC}$ & 64 & 256 & 1,024 & $\mathrm{NC}$ & 64 & 256 & 1,024 & $\mathrm{NC}$ & 64 & 256 & 1,024 \\
\hline Mean Cq & 21.85 & 28.33 & 28.34 & 28.74 & 22.06 & 22.09 & 22.40 & 23.21 & 23.23 & 24.34 & 27.76 & 27.85 & 21.82 & 22.10 & 23.46 & 25.53 \\
\hline SD & 0.07 & 0.18 & 0.25 & 0.09 & 0.01 & 0.02 & 0.17 & 0.07 & 0.20 & 0.09 & 0.05 & 0.08 & 0.01 & 0.04 & 0.14 & 0.01 \\
\hline$\Delta \mathrm{Cq}$ & - & 6.48 & 6.49 & 6.89 & - & 0.04 & 0.34 & 1.16 & - & 1.12 & 4.53 & 4.63 & - & 0.29 & 1.64 & 3.71 \\
\hline \multicolumn{17}{|l|}{ Cleavage } \\
\hline efficiency & - & 98.88 & 98.89 & 99.16 & - & 1.96 & 21.26 & 54.95 & - & 53.70 & 95.67 & 95.93 & - & 17.36 & 67.95 & 92.36 \\
\hline$(\%)$ & & & & & & & & & & & & & & & & \\
\hline
\end{tabular}

The Cq values are described in Supplementary Fig. S1. Cleavage efficiency (\%) was calculated using $\Delta \mathrm{Cq}$ values. 
Table 2. Comparison between SITE-Seq and in silico programs for predicting off-target sites

\begin{tabular}{|c|c|c|c|c|c|c|c|c|c|c|c|c|c|}
\hline \multirow{2}{*}{\multicolumn{2}{|c|}{ Locus }} & \multicolumn{2}{|c|}{ Cas-OFFinder } & \multicolumn{2}{|c|}{ СНОРCHOP } & \multicolumn{2}{|c|}{ CRISPOR } & \multicolumn{2}{|c|}{ CRISPRdirect } & \multicolumn{2}{|c|}{ CRISPR-DT } & \multicolumn{2}{|c|}{ CRISPR-P2.0 } \\
\hline & & NGG & NPR & $\mathrm{NGG}$ & $\mathrm{NP} F$ & NGG & $\mathrm{NPC}$ & $N C F$ & $\mathrm{NDF}$ & NGC & NRF & NCF & $\mathrm{NPC}$ \\
\hline $\begin{array}{c}\text { Off- } \\
\text { target } \\
1 \\
\end{array}$ & $\begin{array}{c}\text { chr1: } \\
18,688,154 \\
\text { (Exon) }\end{array}$ & - & - & - & - & - & - & - & - & - & - & - & - \\
\hline $\begin{array}{l}\text { Off- } \\
\text { target } \\
2 \\
\end{array}$ & $\begin{array}{c}\text { chrl: } \\
33,534,133 \\
\text { (Exon) }\end{array}$ & - & - & - & - & - & - & - & - & - & - & - & - \\
\hline $\begin{array}{l}\text { Off- } \\
\text { target } \\
3\end{array}$ & $\begin{array}{c}\text { chr1: } \\
39,263,530 \\
\text { (Exon) }\end{array}$ & - & + & - & - & - & - & + & + & - & + & - & - \\
\hline $\begin{array}{c}\text { Off- } \\
\text { target } \\
4 \\
\end{array}$ & $\begin{array}{c}\text { chr5: } \\
18,020,045 \\
\text { (3' UTR) }\end{array}$ & + & + & - & - & - & - & + & + & - & - & - & - \\
\hline $\begin{array}{l}\text { Off- } \\
\text { target } \\
5 \\
\end{array}$ & $\begin{array}{c}\text { chr6: } \\
15,796,609 \\
\text { (Intron) }\end{array}$ & - & - & - & - & - & - & - & - & - & - & - & - \\
\hline $\begin{array}{l}\text { Off- } \\
\text { target } \\
6\end{array}$ & $\begin{array}{c}\text { chr10: } \\
10,862,382 \\
\text { (Exon) }\end{array}$ & - & - & - & - & - & - & - & - & - & - & - & - \\
\hline $\begin{array}{l}\text { Off- } \\
\text { target } \\
7\end{array}$ & $\begin{array}{c}\text { chr12: } \\
12,080,780 \\
\text { (Intron) }\end{array}$ & - & - & - & - & - & - & + & + & - & - & - & - \\
\hline $\begin{array}{l}\text { Off- } \\
\text { target } \\
8\end{array}$ & $\begin{array}{c}\text { chr12: } \\
20,542,434 \\
\left(3^{\prime} \text { UTR }\right)\end{array}$ & - & - & - & - & - & - & - & - & - & - & - & - \\
\hline
\end{tabular}

